\title{
Augmented Reality Glasses as an Orientation and Mobility Aid for People with Low Vision: a Feasibility Study of Experiences and Requirements
}

\author{
HEIN MIN HTIKE \\ School of Computer Science and Informatics, Cardiff University, Cardiff, UK
}

TOM H. MARGRAIN

School of Optometry and Vision Sciences, Cardiff University, Cardiff, UK

YU-KUN LAI

School of Computer Science and Informatics, Cardiff University, Cardiff, UK

PARISA ESLAMBOLCHILAR

School of Computer Science and Informatics, Cardiff University, Cardiff, UK

People with low vision experience reduced mobility that affects their physical and mental wellbeing. With augmented reality (AR) glasses, there are new opportunities to provide visual and auditory information that can improve mobility for this vulnerable group. Current research into AR-based mobility aids has focused mainly on the technical aspects, and less emphasis has been placed on understanding the usability and suitability of these aids in people with various levels of visual impairment. In this paper, we present the results of qualitative interviews with 18 participants using HoloLens v1 and eight prototype augmentations to understand how these enhancements are perceived by people with low vision and how these aids should be adjusted to suit their needs. Our results suggested that participants with moderate vision loss could potentially perceive the most benefit from glasses and underlined the importance of extensive customizability to accommodate the needs of a highly varied low vision population.

CCS CONCEPTS - Human-centered computing Accessibility Accessibility technologies•Social and professional topics $\sim$ User characteristics $\sim$ People with disabilities

Additional Keywords and Phrases: low vision, mobility aids, augmented reality, vision enhancement

ACM Reference Format:

NOTE: This block will be automatically generated when manuscripts are processed after acceptance.

\section{INTRODUCTION}

More than 2 million people in the UK are living with a vision impairment or blindness [79]. Their vision impairments can be caused by a variety of underlying visual conditions (e.g., cataract, glaucoma, macular degeneration, diabetic retinopathy, retinitis pigmentosa (RP)) [79], and affect different aspects of visual function such as visual acuity (VA), contrast sensitivity (CS), light sensitivity, colour perception, depth perception, and the visual field (VF). One of the significant problems associated with low vision (LV) is reduced mobility which affects both physical and mental wellbeing of people with $\operatorname{LV}[28,42,49,59]$.

To improve mobility performance in people with LV, it is essential to improve their orientation skills. That is, orientation skills allow people to understand where they are in the environment, where they are going and to plan safe routes to get to a destination. In recent years, several mobility aids based on different technologies have been proposed to improve orientation and mobility $(\mathrm{O} \& \mathrm{M})$ in people with low vision. These aids use various sensors (e.g. 
ultrasonic sensors $[67,82,83]$, depth sensors [15,38,69], cameras $[83,86,87]$, RFID tags $[2,29])$ to gather information about the environment, extract vital information for O\&M and transmit it to people with LV via different modalities: auditory $[1,15,29,82,86]$ and vibrotactile $[15,52,74]$.

While these aids based on alternative modalities are essential for people who are completely blind, the majority of people with LV have useful residual vision [31]; and, despite struggling, prefer to use it to observe the environment [85]. To improve the usefulness of residual vision in people with LV, many mobile applications exist that can manipulate the images to make them more visually accessible such as inverting colours or increasing contrast of photos/videos etc. With advances in augmented reality (AR) head-mounted display systems, there is a unique opportunity to maximise the usefulness of residual vision by providing information in a visually accessible manner. Researchers have proposed visual AR aids to simplify the visual scene (e.g. [24]), to provide distance information (e.g. [30,44]), to extend the size of the visual field (e.g. $[73,95])$, to improve contrast sensitivity (e.g. [34]), to provide magnification (e.g. [72]), and to provide navigational cues in indoor environments (e.g. [9]) and on stairs (e.g. [104]). While these aids were successful in improving some visual functions such as improved clinical measurements (VA, CS, and VF), and obstacle detection and object recognition, there is currently no robust evidence that they can improve mobility efficiency.

The majority of the current research into AR mobility aids providing visual augmentations for people with LV has focused mainly on technical developments such as exploring different computer vision and visualisation techniques and reporting quantitative measures such as mobility efficiency and errors, obstacle detection rates, and clinical visual measurements. Less emphasis has been placed on understanding the usability and suitability of these aids in people with different levels of residual vision and underlying conditions. The majority of the studies did not report participants' opinions and feedback regarding the visual augmentations which determine the success and usability of the aids. Additionally, most of them also failed to involve potential end-users at the initial design stage to capture their specific requirements. Instead, they relied on the researchers' perception of their needs, which may be insufficient due to significant differences between how people with LV and normal-sighted people navigate [98].

To better understand the usability and suitability of visual augmentations in AR for people with varying levels of residual vision, we conducted this qualitative study to understand participants' experiences, preferences, and suggestions for a visual $0 \& \mathrm{M}$ aid. We assessed the perspectives of 18 participants on their experiences with eight prototype visual augmentations using HoloLens v1 (HL) and gathered their feedback and suggestions. The prototypes were developed based on the existing literature, and our prior discussions with people with LV regarding their mobility experiences. The prototypes, described in detail later, performed different functions such as visualising walls; conveying distance information via colours; enhancing details; helping with object recognition; visualising a path for navigation.

Our results suggest that participants with VA better than 2/60 could potentially perceive the most benefit from the prototypes and that different individuals, even with similar levels of residual vision, perceived different benefits and showed different preferences for the prototypes. This suggests that there will not be a one-size-fits-all solution to visual O\&M aids and underlined the importance of extensive customizability to accommodate the needs of the LV population. This paper makes three principal contributions: (i) we provide detailed, qualitative descriptions of participants' perceptions of different types of visual augmentations using AR glasses that complement the existing technically-oriented literature; (ii) we highlight the importance of customizability; (iii) we provide requirements and suggestions to inform the design of the future visual O\&M aids. 


\section{BACKGROUND}

In this section, we provide some definitions of $O \& M$, identify the problems caused by $\mathrm{LV}$, and describe traditional mobility aids, before discussing the related work in the context of technology-based mobility aids, and studies on mobility requirements in people with LV.

\subsection{Mobility and people with low vision}

Mobility, the act or ability to move from one's current location to the desired location in another part of the environment safely, gracefully, efficiently and comfortably [16], is a critical component affecting the quality of life in people with LV [50,51]. Efficient mobility requires good orientation skills, i.e. the ability to use one's vision and/or other senses to understand the local environment at any given time [37]. O\&M in people with LV are influenced by a range of visual factors such as VA, CS, VF, and visual scanning ability [11,27,46-48,53,60,80,91]. For example, the size of the VF together with CS are the most important predictors of mobility performance [27] whereas CS and VA affect obstacle detection and depth perception [80]. Reduced or sudden changes in illumination levels could also compound their mobility problems [46,48,97].

Mobility problems experienced by people with LV differ greatly depending on the nature of underlying vision loss, and problems may include difficulty reading signs, finding the desired destinations, navigating stairs, and avoiding people, obstacles and trip hazards [84,90,91]. These mobility problems can be generalised into two categories: wayfinding (planning and following routes from place to place) and safety (traversing environment safely without tripping or bumping into obstacles) [89].

To alleviate some of the mobility problems, people often use white canes, guide dogs, and other optical aids. White canes are ubiquitous mobility aids, but they are useful only to observe the immediate environment low-lying obstacles [12]. Guide dogs are another popular mobility aid that promotes safety, but their associated training and maintenance costs limit their widespread availability. In terms of optical LV aids, some people with LV use monocular or binocular telescopic devices for some orientation tasks such as reading signs and recognising obstacles at a distance but suffer from usability issues such as difficulty focusing, amplified shakiness from hand and, reduced visual field [54]. Prism lenses are another optical aid that improves the size of VF for people with tunnel vision but introduces problems such as confusion, overlapping views and double vision [6].

\subsection{Technology-based mobility aids}

To overcome the limitations of traditional mobility aids, many technological aids have been proposed to support mobility. Using a wide range of sensors such as ultrasonic sensors $s[67,82,83]$, depth sensors $[15,38,69]$, cameras [83,86,87], RFID tags [2,29], Bluetooth low energy (BLE) beacons [26], GPS [41], these systems assist users with wayfinding [1,26,52], obstacle avoidance $[15,38,43,67,69,86]$, or both $[29,41,81,83,87]$. These aids convey information to users via different modalities: auditory $[1,15,29,82,86]$ and vibrotactile $[4,52,69,74]$. Audio aids provide information with either verbal warnings and instructions $[3,19,58,88]$ or sonification $[7,36,43,61]$ or both [45]. For example, NavCog3 used BLE beacons for localisation and provide turn-by-turn instructions and descriptions of nearby landmarks and points of interest via bone-conducting headphone [81]. Vibro-tactile aids provide feedback through small vibrators embedded in various places such as in the handle of a cane [4], soles inside shoes [96], wrist bracelet [52], vest [18,23], belt [69], and backpack straps [76]. For example, a jacket with eight embedded actuators vibrates in the direction of the obstacles to inform the user of their presence. Besides 
audio and vibrotactile aids, some aids guide the users by steering to avoid obstacles $[13,100]$ or pulling them along the path [8] similar to how a guide dog functions.

In addition to assisting with wayfinding and mobility, some aids help users to improve their orientation skills. Example aids include those helping with reading signs; recognising objects and people; and describing the environment via sonification [7,61]. For example, the vOICe application sonifies the environment by mapping changes in pixel values in images captured by the camera with sound in different tones, pitch, and frequency [7,61].

The primary focus of most of the research into mobility aids has been on people with no vision by providing audio and tactile feedback and therefore overlooked people with LV's ability and preference to use their residual vision.

\subsection{Technology-based visual mobility aids}

As an alternative to sight substitution aids described in the previous section, some research has proposed visual augmentations to improve the usefulness of the residual vision in people with LV using head-mounted displays (HMD). As people with LV have a strong preference to use their residual vision [85], visual augmentations in HMD provide a way of conveying information more intuitively than other sight substitution techniques such as sonification or vibrations in a hands-free manner.

One approach improved the size of VF, an important indicator of mobility performance [53], by overlaying a minified edge or colour image of the wider visual scene onto the centre of the field of view $[14,35,55,57,72,73,95,101]$. It is targeted to people with peripheral vision loss and its impact on mobility performance is yet to be tested extensively. Another study proposed to help people with tunnel vision is an obstacle detection and notification system that tracked and notified the users about dynamic obstacles using dots with different colours and sizes in their central vision, but this has not been tested with participants [102].

However, scene simplification approaches, designed to accommodate the poor visual functions of people with LV and to allow them to focus on obstacles in the scene, have shown to improve safety $[24,30,40,44,78]$. One simplification technique was colour coding different categories of objects by segmenting and classifying them in the image using a neural network to improve visibility $[24,40]$. This visualisation resulted in improved object recognition in static photos [24] but significantly decreased mobility efficiency [40]. Hicks et al. [30] and van Rheede et al. [78] used depth cameras to detect the distances to nearby objects and display them by changing the brightness based on their distances on low-resolution HMDs. This visualisation technique provided depth perception in the simplified scene and resulted in improved obstacle avoidance but increased the time taken to complete the mobility courses [78].

Similarly, Kinateder et al. [44] used HoloLens to simplify and recolour the visual scene based on object distance. The authors reported that four participants with severe vision impairments were able to detect obstacles from further distances and described their qualitative assessments. These simplification techniques showed the potential to improve safety as they helped with obstacle detection and avoidance. However, there is a limited qualitative assessment to understand these simplification techniques' usability for people with different levels of residual visions. Therefore, we included a variation of this technique (prototype 2) in our study to understand how simplified scenes are perceived in people with a wide range of visual levels.

Whilst previous visualisations removed details in the scene by recolouring the whole scene or using lowresolution displays, Angelopoulos et al. [5] proposed a visualisation technique to add depth perception to the existing scene without significantly altering it. Their visualisation conveyed depth information for 6 feet from the 
HoloLens by overlaying coloured wireframes in the visual scene. In an experiment with 10 participants with RP, this visualisation helped to reduce mobility and object grasp collisions significantly but increased the time taken. As this visualisation was limited to 6 feet from the device worn at eye-level, this technique offered limited depth information along the floor (2.71 feet and 3.43 feet ahead on the floor for $1.75 \mathrm{~m}$-tall male and $1.61 \mathrm{~m}$-tall female respectively). This inspired us to include a variation of this technique (prototype 3 ) in our study that extends the depth visualisation to more than 6 feet and to find out how people with different visual conditions other than RP perceive it. Additionally, Zhao et al. [104] proposed visual augmentations to highlight the edges and landings of stairs using HoloLens and 12 participants in the study thought the visualisations to be useful, comfortable and felt secure.

While all these visual augmentations mentioned above have contributed to safe navigation for people with $\mathrm{LV}$, there has been little research on visual mobility aids for wayfinding. Bai et al. [9] used a depth camera and ultrasonic sensors to generate safe-path to follow in indoor environments. They provided audio feedback together with a small directional cue to follow in an HMD. Their study showed improved collision avoidance in ten participants with lazy eyes, but it was not clear if the improvement was due to visual navigational guidance alone. Zhao et al. [105] used HL to provide visual (path, distance and action signs, and destination) and audio (distance and action) guidance of fixed routes in an indoor environment. With 16 participants, their study found no significant effect of visual and audio feedback on wayfinding time, but visual feedback resulted in significantly fewer wayfinding errors and lower cognitive load. In outdoor environments, people with LV rely on wayfinding apps designed for sighted people such as Google Maps [99]. Due to the limited number of studies in visual way finding augmentations, we designed prototype 8 to improve our understanding of how people with LV perceive visual guides for dynamic wayfinding.

In addition to providing visual augmentations to improve obstacle avoidance and wayfinding, other approaches aimed to improve visual aspects important for orientation and mobility. Real-time edge enhancement [55,108] improved CS, whereas digital zooming approaches [56,72] improved VA. While these approaches showed improved clinical visual measurements, how they impact mobility is not known yet. As VA and CS play important roles in mobility, these approaches inspired us to include prototypes 4,5 and 6 which used high-contrast images or edge images to improve CS and allowed dynamic magnification to improve VA and to find out how these enhancements are perceived in people with LV. Other visual enhancements to improve orientation include sign reader [33] and visual cues for search and scanning [107].

Most of the literature, except a few [78,104,107,108], in visual mobility aids did not evaluate the impact of the visual enhancements using participants with varying levels of visual abilities and conditions. Therefore, our understanding of how these visual enhancements impact people with different levels of residual vision is limited. Additionally, only a few studies $[44,104,107,108]$ reported subjective feedback. These subjective data are important to inform future works on how to improve and adapt different visual augmentations to suit the needs of people with LV better.

\subsection{Requirements for technology-based visual mobility aids}

Most of the studies described previously focused on the technical aspects such as visualisation techniques which were designed without the input from potential end-users. While those requirements can be derived from the literature to some extent such as the importance of CS and VF, it is essential to understand the needs and requirements of potential end-users because people use their residual vision in different ways. 
In order to use AR glasses as visual mobility aids, it is crucial to understand how people with LV perceive augmentations in the AR environment. Zhao et al. [103] explored how virtual shapes such as triangles and text were perceived in Epson Monveio glasses with 20 participants with LV. The participants were able to identify shapes and read text on the AR glasses, and the authors recommended guidelines for designing accessible virtual elements for people with LV. However, the study was limited to an indoor environment and involved only basic shapes such as triangles and stars rather than potentially useful visual augmentations.

Two studies observed how participants with LV navigate real-world environments to identify mobility challenges and how they used their residual vision. Szpiro et al. [85] observed 11 participants with LV find a store and purchase an item in the real-world environment. Another study observed challenges that 14 people with LV face while navigating stairs, new buildings and through two city blocks [106]. The authors described requirements for mobility aids such as information needs (e.g. surface-level changes, the direction of changes) and visual requirements (e.g. use of colour and luminance contrast) amongst others.

Some other studies involving participants with LV, explored a broader range of issues in different contexts, through challenges and use of other tools, elicited requirements for their mobility needs and assistive technologies based on other mediums such as auditory and vibrotactile [17,39,62,71,75,98]. For example, Jafri et al. [39] interviewed participants with LV to understand their mobility challenges, their low vision aid usage and to obtain feedback on a depth-based obstacle avoidance system with audio feedback.

The relative lack of studies exploring how people with different levels of residual vision perceive visual augmentations in AR glasses and the limited number of studies on the requirements for visual mobility aids has motivated us to undertake this study. Therefore, in this paper, our aim was to understand if there is an association between the level of residual vision and the preference of different visual augmentations, the perceived benefits and drawbacks of the visual augmentations and to elicit user-based requirements for visual mobility aids by gathering suggestions based on the capabilities of HL and their day-to-day mobility experiences.

\section{METHODS}

\subsection{Participants}

We recruited 18 participants with LV (ten females, eight males) with ages ranging from age 29 to 75 (mean age: 59.3), via local low vision charities (Sight Life, Sight Cymru, and Cardiff University Low Vision Clinic) in Cardiff. To better understand how visual augmentations are perceived by people with different levels of residual vision, we recruited participants with a wide range of visual conditions (Table 1). All participants except two (P3, and P8) were registered as either sight impaired (SI) $(n=4)$ or severely sight impaired (SSI) $(n=12)$. All participants except four completed all the prototype applications. Four (P3, P4, P12, P18) did not complete the navigator, three (P3, P4, $\mathrm{P} 12)$ the object recognition, and one (P12) the zoom overlay prototype. None of the participants had prior experience with AR technology.

Table 1. Participant demographic information. Participants labelled with superscript '6', '7', and '8' did not complete zoom overlay, object recognition and navigator prototypes respectively.

\begin{tabular}{lllcccc}
\hline ID & $\begin{array}{l}\text { Age/ } \\
\text { Gender }\end{array}$ & $\begin{array}{l}\text { Registration } \\
\text { status }\end{array}$ & Diagnosis & $\begin{array}{l}\text { Binocular } \\
\text { Visual Acuity }\end{array}$ & $\begin{array}{l}\text { Binocular } \\
\text { Contrast } \\
\text { Sensitivity }\end{array}$ \\
\hline P1 & $64 / \mathrm{F}$ & SSI & Retina Dystrophy & $6 / 60$ & Significant loss & $\begin{array}{l}\text { Moderate } \\
\text { contraction }\end{array}$ \\
\hline
\end{tabular}




\begin{tabular}{|c|c|c|c|c|c|c|}
\hline P2 & $38 / \mathrm{M}$ & SI & Horizontal Nystagmus & $6 / 36$ & Noticeable loss & $\begin{array}{l}\text { Moderate } \\
\text { contraction }\end{array}$ \\
\hline $\mathbf{P} 37,8$ & $58 / \mathrm{M}$ & None & $\begin{array}{l}\text { Cataract, Macular } \\
\text { Degeneration }\end{array}$ & $6 / 48$ & Noticeable loss & Full-field \\
\hline $\mathbf{P 4} \mathbf{4}^{7,8}$ & $61 / \mathrm{M}$ & SSI & $\begin{array}{l}\text { Macular Degeneration, } \\
\text { Myopia }\end{array}$ & $6 / 48$ & Significant loss & $\begin{array}{l}\text { Moderate } \\
\text { contraction }\end{array}$ \\
\hline P5 & $69 / F$ & SSI & $\mathrm{RP}$ & $1 / 40$ & Severe loss & $\begin{array}{l}\text { Central vision } \\
\text { loss }\end{array}$ \\
\hline P6 & $65 / F$ & SSI & $\begin{array}{l}\text { Cataract, } \\
\text { Detected retina, Myopia }\end{array}$ & $3 / 60$ & Significant loss & $\begin{array}{l}\text { Moderate } \\
\text { contraction }\end{array}$ \\
\hline P7 & $67 / M$ & SI & Glaucoma, Cataract & $3 / 60$ & Severe loss & $\begin{array}{l}\text { Moderate } \\
\text { contraction }\end{array}$ \\
\hline P8 & $62 / \mathrm{M}$ & None & Nystagmus, Myopia & $6 / 18$ & $\mathrm{Ok}$ & $\begin{array}{l}\text { Moderate } \\
\text { contraction }\end{array}$ \\
\hline P9 & $47 / F$ & SSI & Myopia & $6 / 90$ & Significant loss & $\begin{array}{l}\text { Moderate } \\
\text { contraction }\end{array}$ \\
\hline P10 & $48 / \mathrm{F}$ & SSI & $\begin{array}{l}\text { Wet macular } \\
\text { degeneration, } \\
\text { Stargardt's disease }\end{array}$ & $1 / 40$ & Severe loss & $\begin{array}{l}\text { Moderate } \\
\text { contraction }\end{array}$ \\
\hline P11 & $29 / \mathrm{F}$ & SSI & $\begin{array}{l}\text { Myopia, Nystagmus, } \\
\text { Cone dystrophy }\end{array}$ & $6 / 60$ & Significant loss & $\begin{array}{l}\text { Moderate } \\
\text { contraction }\end{array}$ \\
\hline $\mathbf{P} 12^{6,7,8}$ & $59 / \mathrm{F}$ & SSI & $\begin{array}{l}\text { Junior macular } \\
\text { degeneration }\end{array}$ & $3 / 60$ & Significant loss & $\begin{array}{l}\text { Central vision } \\
\text { loss }\end{array}$ \\
\hline P13 & $46 / \mathrm{M}$ & SSI & - & $1 / 40$ & Severe loss & Profound loss \\
\hline P14 & $72 / \mathrm{M}$ & SSI & $\begin{array}{l}\text { Nystagmus, } \\
\text { Congenital optic } \\
\text { atrophy }\end{array}$ & $6 / 60$ & Significant loss & $\begin{array}{l}\text { Moderate } \\
\text { contraction }\end{array}$ \\
\hline P15 & $70 / F$ & SSI & $\begin{array}{l}\text { Glaucoma, Cataract, } \\
\text { Nystagmus, Aphakia }\end{array}$ & $6 / 90$ & Significant loss & $\begin{array}{l}\text { Moderate } \\
\text { contraction }\end{array}$ \\
\hline P16 & $70 / \mathrm{M}$ & SSI & $\begin{array}{l}\text { Myopia, Cataract, } \\
\text { Glaucoma, Detected } \\
\text { retina }\end{array}$ & $1 / 32$ & Severe loss & $\begin{array}{l}\text { Moderate } \\
\text { contraction }\end{array}$ \\
\hline P17 & $75 / F$ & SI & $\begin{array}{l}\text { Vision loss caused by } \\
\text { stroke }\end{array}$ & $6 / 12$ & Noticeable loss & $\begin{array}{l}\text { Moderate } \\
\text { contraction }\end{array}$ \\
\hline $\mathbf{P}^{18}{ }^{8}$ & $67 / F$ & SI & $\begin{array}{l}\text { Vision loss due to brain } \\
\text { damage }\end{array}$ & $6 / 12$ & Noticeable loss & Full-field \\
\hline
\end{tabular}

\subsection{Procedure}

This study was conducted between October 2019 and March 2020. The study consisted of a semi-structured interview that lasted approximately one hour, and the interview was voice recorded. The study environment consisted of a well-lit room comprising of tables, chairs, and various items (such as books, water bottles, cups, laptops, photos) placed around the room, and a corridor with a few turns. Before starting the session, we scanned the environment with the HL so that it could generate the spatial information necessary for prototypes quickly and recognise the participants' current location in the environment. We also marked a destination point at the end of a corridor for the navigational task.

At the beginning of the interview, we asked each participant about their background information such as demographics, underlying visual conditions, registration status, and LV aids use. We then measured VA and CS using 
the ETDRS LogMAR and Pelli-Robson contrast sensitivity charts respectively and conducted a confrontational visual field test. We did not measure participants' colour perception however, we asked them to self-report any other visual impairments they are aware of. After collecting background and visual information, we fitted the participants with HL to demonstrate the prototypes.

During the demonstration process, we showed one prototype after another and explained their intended purposes. For each prototype, we presented all adjustable options such as colours or line sizes to the participants and asked them to observe the environment through the augmented views. If they were comfortable to do so, they were encouraged to get up and walk around the room to experience changes in the visual augmentations while they were mobile. For prototype 8, we asked the participants to get up and walk out of the room following the path shown in HL to reach the destination. To maintain consistency across participants, the interview guide consisted of the following questions for each prototype: (i) describe what you are seeing; (ii) anything that you find useful, and why; (iii) anything you find disadvantageous for your vision, and why; (iv) how to improve. At the end of the session, we asked what their favourite prototypes were, what enhancement they want to see on AR glasses for their mobility problems, and what they think of the device itself. The ethics committee of the School of Computer Science and Informatics, Cardiff University approved this study (approval no: COMSC/Ethics/2019/032).

\subsection{Analysis}

The voice recordings were transcribed in verbatim. The first author read and re-read the transcripts and wrote memos of early impressions. Then the transcripts were uploaded to NVivo12 for data handling and coding. We used deductive thematic analysis with open coding, where codes were developed and updated throughout the process. The codes were then merged into themes describing the patterns in the data relevant to the research questions. The impressions from the data, themes and coding progress were discussed regularly as a team. Eventually, we analysed the data individually for each prototype as the overlapping themes across eight prototypes failed to provide insightful details due to significant differences between the purposes of prototypes.

\subsection{Device}

We used HoloLens v1 [63] from Microsoft to demonstrate the AR prototypes to the participants. HL is a see-through $\mathrm{AR}$ device with an approximate field of view of $30^{\circ} \mathrm{H}$ and $17.5^{\circ} \mathrm{V}$. It includes one depth camera and four environment understanding cameras to capture the state of the surroundings and one 2MP camera for photos and $30 \mathrm{fps}$ videos. Users can interact with the AR environment using voice command, hand gestures, or a dedicated Bluetooth clicker. HL was chosen for this project for its ability to be worn with eyeglasses. 

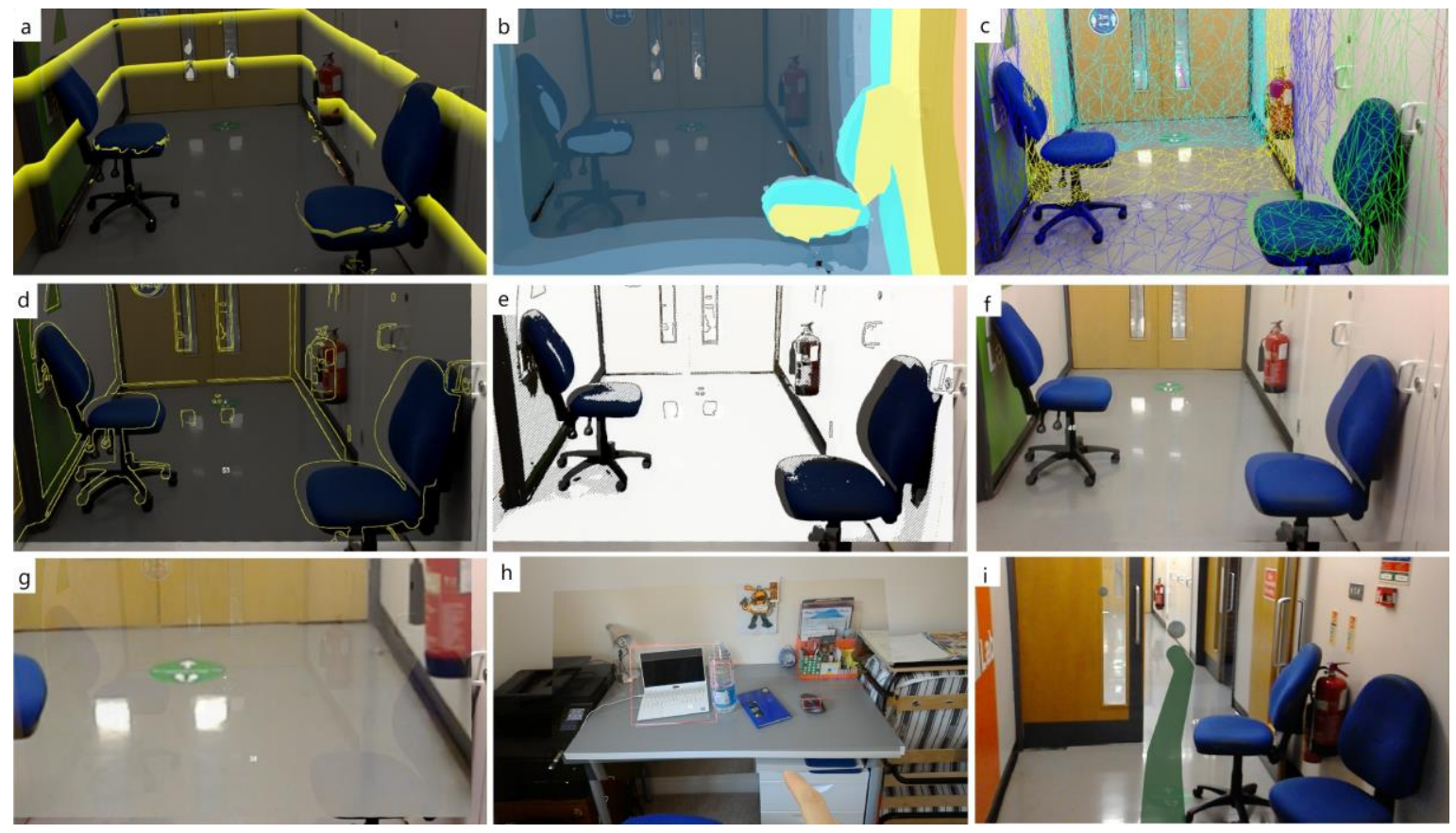

Figure 1: Screenshots of prototypes; (a) lines on walls; (b) colour overlays; (c) coloured-line overlays; (d) edge overlays; (e) comic overlays; (f) live view overlay; (g) zoom overlay - zoomed view taken from the same location as ( $\mathrm{f}$ ), (h) object recognition, (i) navigator. Darkened images in (a) and (d) of the screenshots were caused by black pixels which were rendered transparently in in-device view. In images (d), (e), and (f), taking screenshot from monocular view exacerbated misalignment of overlays and real-world environment compared to the actual binocular view through $\mathrm{HL}$.

\subsection{Design Procedure of AR Prototypes}

This study represents the third phase in our project that aims to develop an effective mobility aid for people with $\mathrm{LV}$ and to evaluate its effectiveness quantitively with mobility trials. The first phase of our project, a systematic literature review on how current visual augmentations in HMDs affect mobility in people with LV [32], highlighted the lack of user involvement in the design and development stages of the aids, and the lack of qualitative data in most of the existing literature. To better understand the requirements for visual mobility aids in people with LV, we undertook four focus groups with 20 participants with LV as the second phase of our project. In the focus groups, we discussed the unique mobility challenges that the participants experienced in their day-to-day activities, the techniques they used to cope with them and how they want technology to help alleviate those problems. Some problems participants discussed in the focus groups included difficulty detecting obstacles, walls and surface level changes, being uncertain of where they were and difficulty orientating themselves when travelling in unfamiliar environments and knowing the distances to objects due to reduced depth perception and contrast sensitivity.

In this study, we created eight prototypes (Figure 1) based on the results of our systematic literature review and focus group discussions (please see the companion video produced to demonstrate each prototypes). These prototypes served six different purposes: to visualise walls and objects with large enough vertical surfaces (prototype 1); to improve depth perception (prototypes 2 and 3); to provide edge and contrast enhancements (prototypes 4,5); to provide dynamic magnification (prototype 6); to showcase the device's capabilities of object recognition (prototype 7); to provide visual navigation cue (prototype 8). We developed prototype applications for 
HL using Unity 2018.5.7f1 [92]. Software packages used included Mixed Reality Toolkit 2017.4.3.0 [66], OpenCV for Unity [22], and YOLOv3-Lite [77].

\subsubsection{Prototype 1 - Lines on Walls}

Some participants in our previous focus group work reported difficulties identifying walls in their environments and walked into them due to reduced contrast sensitivity. To alleviate this problem, we drew inspiration from LV clinics and hospitals, where it is a common practice to paint high-contrast colour lines on the walls to help people with LV with tracing [20], recognising where the walls are and assist them with orientation and wayfinding. In order to explore the effects of similar virtual lines in an AR environment, we created this prototype to draw lines on the vertical surfaces using Microsoft HoloToolKit [64]. The application drew four lines per one meter of a vertical surface, and the width of each line was $0.1 \mathrm{~m}$. There were four options for the line colour: red, green, blue, and yellow. The lines are drawn in real-time in a pre-scanned environment, or with a delay of a few seconds in an unknown environment.

\subsubsection{Prototype 2 - Colour Overlays}

People with low vision experience reduced depth perception [80]. Due to their inability to perceive distance, most of the participants in the focus groups experienced problems such as difficulty navigating stairs or missing a step, tripping on obstacles due to the inability to distinguish how far away they were, falling due to the inability to perceive surface level changes. This prototype aimed to improve depth perception for people with LV. Using a variation of the colour-based distance representation in an experiment with four participants with LV [44], this prototype mapped warmer colours to nearer distances and cooler colours to further away distances and overlaid the solid colours in the scene. This distance to colour mapping was arbitrary, and the prototype used ten different colours in total. The first colour covered the distances between $0.3 \mathrm{~m}$ to $1.5 \mathrm{~m}$ and the last colour covered distances beyond $3.5 \mathrm{~m}$. The eight middle colours covered $0.25 \mathrm{~m}$ distances each. At one time, the maximum total volume of environment painted with colours was $10 \mathrm{~m}^{3}$. We decided to include this prototype in our study to further improve our understanding of how this simplified colour-based visualisations of distances was perceived by people with a wide variety of visual conditions.

\subsubsection{Prototype 3 -Coloured-line Overlays}

Like the previous prototype, this aimed to provide improved depth perception for people with LV. Increasing the amount of visualisation from a previous study [5], which visualised the environment up to 6 feet from the user, this prototype visualised everything that had been scanned by the HL. Instead of drawing solid colour overlays like prototype 2, this prototype used spatial mapping renderer [93] from Unity to draw triangular mesh on top of the real-world environment, and the mesh was coloured based on the distance to the usser. Each one-meter distance from 1 to $10 \mathrm{~m}$ was coloured by nine different colours (red, green, ..., orange) and white colour was used to denote distances beyond $10 \mathrm{~m}$. This application was configured to draw a triangular mesh made up of approximately 1000 triangles per $1 \mathrm{~m}^{2}$ area.

\subsubsection{Prototype 4 - Edge Overlays}

As CS together with VF size are important predictors of mobility performance in people with LV [27,80], most participants in our focus groups reported experiencing problems that stemmed from reduced CS such as the distinguishing obstacles and walls in the environment, and identifying different objects and their boundaries. In order to highlight distinctive objects in the environment by providing edge enhancements, we developed this prototype to overlay edges in the users' view in a similar fashion to this Google Glass application [34]. This 
prototype used the video camera of HL to capture the environment and calculated edge images from the video feed using Canny edge detection implementation of OpenCV [70]. Then the edge images were displayed, approximately $1.5 \mathrm{~m}$, in front of the user in real-time at 30fps. Due to well-documented error in the un-distortion function of HL's image processing pipeline [65], there was a slight misalignment between edge images and their real-world views. The participants could adjust the colour (red, white, green, blue, and yellow), and the size of edges.

\subsubsection{Prototype 5 - Comic Overlays}

To alleviate problems caused by poor CS as described in previously, this prototype aimed to provide high-contrast views to people with LV by overlaying a comic-looking image of the environment in real-time. This prototype, based on the comic filter from OpenCV for Unity plugin [22], generated grayscale, high-contrast images of the environment. Like the previous prototype, filtered images were displayed at approximately $1.5 \mathrm{~m}$ in front of the user at $30 \mathrm{fps}$. Similar issue with image alignment was present in this prototype.

\subsubsection{Prototype 6 - Live-view and Zoom Overlays}

In our focus groups, some participants described occasions where they observed their environment by taking photos on their mobile phones and viewing them by zooming in to different parts of the photos on the phone screen. To provide a similar functionality on HL, this prototype displayed either the video feed of the camera from HL without additional manipulations (live-view overlays) or the zoomed view of the video feed (zoom overlays) in realtime. Like prototypes 4 and 5, the images were displayed at approximately $1.5 \mathrm{~m}$ in front of the user at $30 \mathrm{fps}$ and have a slight image misalignment problem. This prototype also allowed users to magnify the centre of the visual scene using voice command ("zoom in", "zoom out") while they were stationary and the zoom level reset when users started moving. During the magnified view, the real-world background remained visible and unaligned.

\subsubsection{Prototype 7-Object Recognition}

We included this prototype to demonstrate some possible machine learning capabilities of HL. Using click gesture or Bluetooth clicker, users could analyse the objects in the scene. The prototype returned the results with both visual (red squares around identified objects in images) and audio feedback. Making a new request to analyse the scene removes the previous visual feedback to avoid confusion. The object recognition was performed on an external computer to which the HL was connected remotely. The YOLOv3-Lite algorithm, trained to recognise 80 classes (e.g. dog, cup, laptop, book, chair, person) [77] was used for recognition tasks.

\subsubsection{Prototype 8 - Navigator}

To help people with LV with navigation and orientation in unfamiliar environments, this prototype dynamically generated a path from user's current position to a predefined destination and visualised the path by drawing a green line on the floor. Upon command, we used the NavMesh API [94] from Unity to generate the traversable path. The properties of a walkable area could be defined in NavMeshAgent class, and we used $0.4 \mathrm{~m}$ radius, $1.7 \mathrm{~m}$ height and $0.5 \mathrm{~m}$ step height in our prototype. This prototype could be used in conjunction with all the other prototypes except prototypes 2 and 3.

\section{RESULTS}

This section begins by describing the three themes that emerged across the eight prototypes. The main theme that emerged from this study was the need for extensive customizability of the visual augmentations. Many participants reported perceiving the visual augmentations differently from one another (e.g. different colours, wavy or curvy lines, blurriness) and made suggestions to accommodate their visual needs. Their suggestions included altering the 
sizes, colours, locations and the number of virtual elements provided by the visual augmentations. The second theme was that visual augmentations could provide an improved understanding of the environment and obstacles. The majority of the participants noted that improvement in obstacle detection was one of the main advantages during the demo session. Both highlighting objects using holographic overlays in the real-world environment (prototypes 1, 2, 3, 8) and overlaid two-dimensional filtered images (prototype 4, 5, 6) improved their awareness of objects and obstacles and allowed them to understand the layout of the environment better. Consistent virtual elements in the augmented views also served as depth cues, allowing them to know the distances of obstacles better. They also felt that the visual augmentations lit up the environment, and it allowed them to see better. The last theme was the technical weakness of current hardware and software implementation. The technical issues reported by participants included slow and sparse depth acquisition, video delay, and misalignment and instability of filtered image overlays.

In addition to the three themes across the eight prototypes, we observed some important points. Real-time filtered images in prototypes 4, 5, and 6 improved participants' ability to perceive details in the visual scene such as improved face recognition and enabled them to see details in images. They were also generally enthusiastic about having help in recognising objects and having visual navigator. They also felt occlusion caused by persistent visual enhancements was a problem in observing details in the environment. Other comments regarding visual issues were difficulty seeing colours properly and excessive brightness from augmentations.

While the eight visual augmentations used in this study shared some similarities, the differences between each unique augmentation are more significant. Additionally, one of the aims of this study is to understand how people with LV perceive different types of visual augmentations. Therefore, we will describe the results for individual prototypes for greater understanding in the following sections.

\subsection{Lines on Walls}

Seven participants (P2, P3, P4, P6, P10, P11, P13) said they liked this prototype and agreed that this improved the overall usefulness of their residual vision in different ways such as improved spatial understanding and depth perception.

While the primary purpose of this prototype was to visualise the walls, only P13, who could not see the walls with his normal vision, felt this was beneficial for that specific purpose. As P13 said, "I can't see the wall, but I can see the green, ... It lets me know where the wall is". For the rest of the participants, this prototype was not helpful to visualise the walls as they did not have trouble seeing them. As P1 said: "It doesn't help because I can see the walls anyway".

However, eight participants (P2, P3, P4, P6, P8, P10, P11, P10) found this prototype to be helpful to understand the environment and obstacles better. Four participants (P2, P3, P4, P11) felt that this enabled them to be more aware of the obstacles in the environment as the lines drawn on the vertical surfaces of the obstacles were easy to perceive. "It gives me a little bit more idea that there's something there." (P3) and "The chairs come out because there's light on it and it reminds me that something is on the way" (P11).

As the lines are drawn at fixed distances from the floor, changes in the height of the lines enabled P2 and P4 to deduce the layout of the environment better. "... looking down the skirting board there, the shape of green has changed, and it is sort of sloping down. I am assuming it is just a slope and [I am] adjusting ..." (P4). Changes in the number and the size of the lines visible in HL also served as depth cues for six participants (P3, P6, P8, P10, P11, $\mathrm{P} 12)$ to gain an improved sense of depth perception. "It gives you a good idea of how far the wall is from you" (P11), 
and "The further away, the bigger area it has... it can give you depth perception" (P12). Additionally, four participants (P3, P4, P6, P10) also thought that these augmented lines were useful in a dimmed or a dark environment to light up the scene. "That helps absolutely. I think it could be very useful ... I didn't expect it to work in the dark ... looking around, I know there's a chair immediately" (P4) and "It lights up things more for me" (P10).

Despite the benefits described above, some technical and visual issues with this prototype limited its usability for many participants. Technical issues associated with this prototype stemmed from slow and limited depth sensor of the HoloLens. Five participants (P2, P3, P4, P18, P12) noted that the visual augmentations did not update quick enough when they moved. "The slow update is a problem, the leftover depth images" (P3) and "another problem I've got is that I am seeing an after image." (P12). Seven participants (P3, P4, P6, P8, P10, P15, P18) were able to perceive small discontinuities in the lines caused by holes in the depth maps. "I can see patterns on it" (P18) and "There are green specks coming out here" (P6).

Additionally, the visual issues associated with this prototype included occlusion caused by the lines, and difficulty perceiving the visual augmentations. For four participants (P9, P12, P15, P16), the presence of the augmented lines in their vision was a source of confusion or distraction. As P16 and P12 said: "It is just confusing me now ... with all the lights [from augmentations]. I am not sure what I am looking at" and "... because you are concentrating on that [lines on walls], you forget what's around you". Five participants (P3, P4, P11, P6, P12) disliked that the visual augmentations were occluding the real-world environment. As P3 and P11 said: "You were trying to look at things on the walls, then it wouldn't be good. The green light comes across it" and "If somebody sat there, you are only going to see it behind them".

Four participants (P6, P10, P12, P18) also had trouble perceiving the colours of the lines correctly. They mentioned that they saw the visual augmentations in many different colours at the same time although the prototype used only one colour at a time and described their experiences as "psychedelic" (P12), and "they're different colours, the shades are changing" (P6). P10 and P17 also expressed concerns about the brightness produced by the visual augmentations: "because I can see a lot of brightness, it might blend in together [with environment] and the glare might be too much for my eyes" (P10).

Other important limitations of this prototype as described by some participants were its inability to distinguish doors and walls (P4, P5, P12) and its failure to pick up and visualise obstacles without large enough vertical surfaces or glass surfaces (P4, P11, P12).

\subsection{Colour Overlays}

Only three participants (P6, P11, P17) out of 18 felt this prototype could be useful in improving their residual vision. For them, overlaying multiple solid colours in real-world scene provided better-defined shapes of the obstacles and made them seem closer for better understanding of the environment and obstacles in it. "It looks clear, it looks as though it's defined" (P6) and "There is no blurring or zigzagging around the objects, they are not foggy" (P17).

Although seven participants (P3, P6, P8, P10, P11, P12) agreed that using solid colour overlays to indicate distance information helped them to improve their depth perception, its drastically unfamiliar visual representation and occlusion of real-world environment rendered this prototype unsuitable as a visual mobility aid for most participants in this study.

For nine participants, this visualisation of distance as colours was too unintuitive. They described their experience as "psychedelic" (P8, P4), "abnormal" (P18), "funny shapes" (P1), "prism" (P6), "confusing" (P7, P16), "weird" (P12, P10), "lots of reflection" (P10), "modern art installation" (P8), "dunes in the desert" or "being on drugs" 
(P12). Four participants (P10, P11, P13, P14) mentioned their difficulty in perceiving colours correctly: "I see blue, red, green and a colour between red and green only" (P14), and "... that would be useful if we get the colours right" (P10).

Seven participants (P2, P4, P7, P11, P15, P16, P18) voiced their concerns about not being able to see the details in the environment as the prototype was overlaying the scene with solid colours. "I worry about not being able to see them [obstacles]" (P18), and "I can't make anything out, it obscures all of you. It's concentrating on something, and that's the worst thing, you could fall over" (P16).

\subsection{Coloured-line Overlays}

Only four participants (P2, P10, P11, P18) said they liked this prototype and felt this to be useful to judge the distance. For other participants, they found some aspects of this prototype to be useful even if its overall performance was not good enough.

For three participants (P2, P4, P5), coloured lines drawn on the walls and floors and objects allowed them to see further ahead than what they could see with their normal vision and therefore improve their understanding of the layout of the environment. "It gives [me] a sense of how big the environment is ... it's very cool" (P2), "it's taken me ten meters in advance... so I can sort of work out with that" (P4), "Because I can only see things each side ... then I know that it's that gap that I have to walk through" (P5). Four other participants (P6, P10, P11, P15) felt that the lines provide better-defined boundaries to the objects and obstacles. "it is good because the shape is standing out" (P6), "that's much better ... because I can actually see the edge of things" (P11), and "it helps me know something is there because it separates [objects]" (P10).

However, six participants (P4, P5, P9, P10, P11, P13) with self-reported poor colour perception had a difficult time understanding the distances in this prototype due to the use of 11 different colours. "I can't make out the colours, they are wrong for me" (P4), "I can't see the colours, I can only just see the lines" (P11), and "all the colours are just one for me. It doesn't appear to be any depth perception there" (P9).

Due to having a large number of lines ( 1000 triangles per $1 \mathrm{~m}^{2}$ ) for $10 \mathrm{~m}$ ahead at any given time, four participants (P4, P9, P12, P15) felt that the prototype was providing an overwhelming amount of visual information, leading to information overload. "It worked perfectly in a small, confined area [in room], but now [in corridor] it is uncomfortable because it's focusing [showing] too far away and I don't need to do [know] that presently" (P4), "I don't think I can walk like this ... too much visual information" (P15), and “... there are a lot of patterns, it's very busy, and you can't make out what's there" (P12).

Six participants (P1, P3, P8, P11, P17, P18) also disliked that the lines were obscuring parts of the real-world environment which prevented them from reading signs or identifying objects. "I can't see anything really, all I see is yellow lines" (P17), "I am losing information that I get from my normal vision of what is here" (P8), and "when you were stood there, I couldn't even see you behind all those stripes" (P1).

Other significant limitations of the prototype experienced by participants included slow updates (P8), not recognising glass surfaces due to depth sensor limitation (P11), and lack of object boundary between two objects with similar distances (P3). 

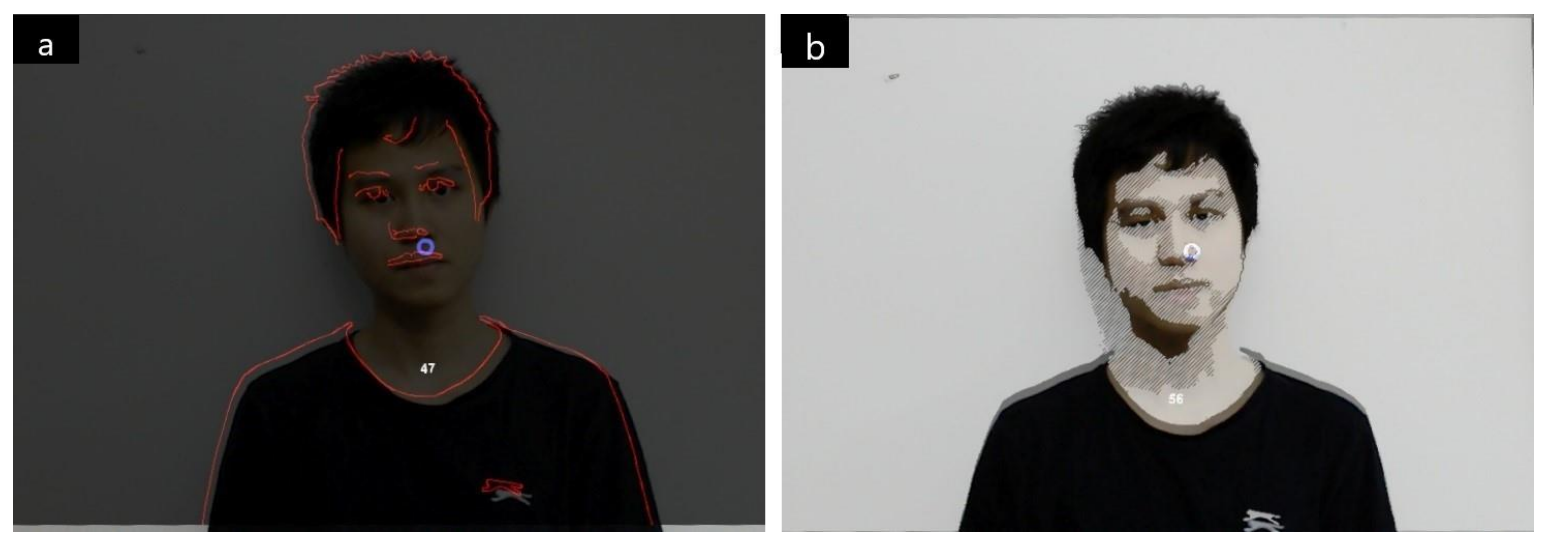

Figure 2: Edge and comic overlays on a face; (a) example of sparse information, missing edges on chin, neck, and hairline; (b) comic overlay on a face. Darkened image in (a) is caused by black pixels that are transparent in in-device view of HL. Misalignment in overlays exacerbated by capturing screenshot in monocular view instead of actual binocular view of $\mathrm{HL}$.

\subsection{Edge Overlays}

Eight participants (P2, P3, P4, P6, P9, P10, P12, P17) agreed that overlaying edge images improved the usefulness of their residual vision. The edge images allowed them to see obstacles and objects better (P2, P4, P9, P17), read signs (P12), see facial expression (P4), and see some details in images (P1, P4, P6). "I can see that there's a chair there ... that's something I can't see normally" (P2), "It can't show me the walls but show me the writings. I can read [letters on the wall], it's more recognisable" (P12), "if I was sat in a pub and I wanted to see your facial expression I think that might work" (P4), and "it's a building? Wow, I am just amazed that I can identify the picture from a distance, ... Brilliant! Excellent! (P6).

Nevertheless, one fundamental visual issue with this prototype was that the information provided by the edges alone was too sparse to understand the scene (Figure 2). Seven participants (P1, P2, P3, P5, P7, P11, P16) felt that although the edge overlays allowed them to see that something was there, it was not enough to make out what they were seeing. "I can see lights on your arms. It is like an X-ray, ... I wouldn't be able to distinguish what it was" (P5), "The lines don't make a difference. Your face is not clear anyway, ... I can't tell you what [object] it was on" (P7), and "I am not sure, I can see outlines of something, but I don't know what it is" (P16). Additionally, P2, P8, P18 noticed lack of depth perception in the augmented view as the prototype overlaid the 2D edge images on the scene. "It's much closer than it is actually. That's what I am finding somewhat confusing" (P8), and "I am seeing it [handbag] much nearer. I am not seeing the distance between them" (P18).

The main weakness of this prototype as described by half of the participants (P3, P5, P6, P7, P9, P11, P12, P15, P16) was a technical issue caused by unstable edge images. As edge images were extracted at 30fps without stabilisation, slight movements of the head could result in different edges being extracted and displayed for each frame leading to slightly jumpy and moving edges. "It is constantly updating ... I think after a while, it is starting to take up awful lot, from the view to my brain" (P3), "It's picking them up all and that's what's flashing a lot" (P9), and "if you move your head, it's dizzying” (P6). 

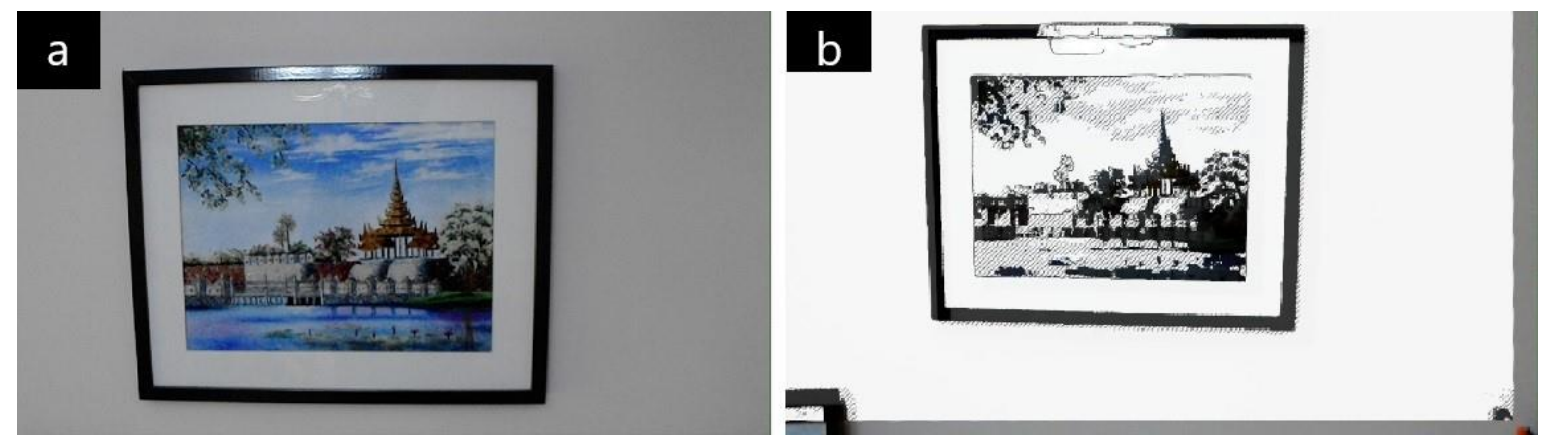

Figure 3: View of a painting; (a) normal, (b) comic overlay

\subsection{Comic Overlays}

Eight participants (P2, P3, P4, P6, P10, P11, P12, P17) liked this prototype and felt it improved their residual vision. Six participants (P2, P6, P10, P12, P17) felt that high-contrast black and white overlays enabled them to perceive facial features better (Figure 2). "I like the way it emphasises facial features and facial expressions ...[it] could help with face recognition" (P2), "Oh my goodness, that's definitely better. Wow! I can see eyebrows and everything" (P6), and "I can see your face clearly, wow!" (P10). This prototype also enabled six participants (P4, P6, P8, P10, P11, P15) to observe significantly more details in images and paintings (Figure 3). "This simplify things a bit. These are quite good. ... it clarifies the overall shape of those three pictures." (P8), and "I can see more details in brush [on a picture], I can see there are bristles on the brush" (P11). High contrast overlays also made it easier to see objects and obstacles for P2, P3, P11, and P15. "It makes things stand more, definitely" (P15), and "if you look around, it emphasises different things" (P2).

However, six participants (P2, P3, P4, P5, P7, P16) commented that the overlays were not stable enough. This instability of images limits the participants' ability to use this prototype for extended periods or their ability to comprehend the visual scene. "This might give me a bit of headache if I were to put this on for a long period of time" (P2), "If you are wearing for a long time, it needs to be stable. Coz the wall outside the window is redrawing itself." (P3), "There's something moving on there, a lot of circles or something like that" (P16). Some participants also noted that this visual augmentation is difficult to perceive at times because of blurriness due to slight misalignment of overlays and real-world (P8, P18), brightness due to large portion of the overlaid image being white (P3 and P1), and slight video lag (P2, P3).

\subsection{Live-view and Zoom Overlays}

This prototype offered normal and magnified views of the scene. In the normal view, viewing the environment through the overlaid images improved colour perception for four participants (P4, P7, P9, P11). "I can see you a lot clearer ... there are a lot of colours down the wall, it's a lot clearer..." (P7), and "Everything is a bit brighter and clearer... red seems a lot brighter to me, but I haven't been able to see red before ..." (P11). Five participants (P4, P7, P8, P9, P14) remarked that they were able to perceive a lot more details in images due to improved colours, sharpness and contrast and closeness of the two-dimensional overlay image compared to the real-world distance. "When I am using this, the buildings [in the photo] are separate... I could see more details on the pictures." (P8), "If I look at it [photos], I have no idea at all what's on the inside. Yes, that's very good" (P4).

Five participants (P4, P6, P9, P11, P17) also agreed that zooming up to a certain level was a useful feature of this prototype. "If I look at that and all I see is a dark space behind the glass, and yet I was able to see things happening 
behind it. Wow! That's impressive" (P4), and "I can see what's in the cabinet now" (P17). P6 was even able to read a few sentences from the participant information sheet using this prototype.

However, as the zoomed image is overlaid on top of real-world, it made it hard to see the details clearly as the real-world background was visible resulting in double and blurry vision (P1, P3, P7, P11, P16, P18). "Seems to be giving me [a] sort of multi-vision or double vision. Everything is sort of double vision, that is even if I close one eye. It is not clear at all" (P3), "The blurriness of double vision is taking it [improved brightness and definition] away from that" (P11).

\subsection{Object Recognition}

Nine out of 15 participants who tried this prototype said this could be useful for them in their day-to-day lives. As P6 said: “Wow! Fantastic! That would be brilliant. It's like somebody telling you what you see”. Four participants (P7, P10, P11, P18) felt this prototype could help them search and locate items of interest in home and supermarket environments. "If you have a desk or table with lots of objects on there, I will be able to search [using this prototype]. It could help in the supermarket too" (P10), and "When I am out shopping, I can't see everything. So, having something to tell me what was there, I'd know then" (P18). P11 and P16 also noted knowing what things are in advance could be helpful to them. "I like it. Most of the times, looking at stuff, in my head, I am imagining what it is, and when you get close to it, it could be completely different" (P11).

Six participants (P1, P5, P8, P13, P14, P17) who disliked this application did so for a variety of reasons. Four participants (P5, P8, P14, P17) felt that their vision was enough to identify and recognise objects. "I can see things in front of me naturally anyway... I don't think I can identify things with this view" (P5), and "I don't think so [it is not useful]. Like I said, at this present time, I think I'm coping well" (P17). P13, on the other hand, was unable to see objects in both real-world and in the HL highlights due to his severe vision loss. P1 did not believe these recognitions were robust enough to be useful in her daily life: "The question is they don't always work".

Suggestions to improve the usefulness of this prototype included face recognition (P1, P2, P8, P9, P11), providing environmental information (P15, P16) such as doors and road signs, and recognising only the targeted area where a person is viewing (P2).

\subsection{Navigator}

Ten out of 14 participants who completed the demonstration for this prototype liked it. They were able to follow the line smoothly and felt more confident that they were going in the right direction while following the line. "Knowing the green line shows you the gap that you can walk through, [I] can walk confidently" (P2), "It is useful for me to navigate if you know where you need to go and it'll tell you go there, turn left, right. That will be good to find a way around as long as they have a plan to go around" (P6), and "Confidently, having something that's just going to take me through a building and say stop there, you are at the right room or at your location would help me" (P8).

Although this prototype was well-received by the majority of the participants, there were some areas of improvement for better usability. One drawback of this prototype was constantly updated shortest path. As it constantly recalculated the shortest path to the destination, P7, P10 and P11 felt the line moved too much and was at times too close to the walls or too jagged. Additionally, we found that participants have different preferences for the positioning of the line. Some were happy to have the line drawn on the floor (P10, P11), whereas some want it to be higher up at waist level (P8, P15), or on the wall (P17).

Out of four participants who did not like this prototype, three (P1, P5, P13) of them had difficulty seeing the line on the floor due to their relatively poorer residual vision. P16, however, felt uncertain that the line would take him 
through safely. He described his uncertainty as "It's a lot of work. When I follow that line, but you still got to fear for things around you, people sat there and that. To pass a chair, for instance, would the green line go over the chair? Would the line stay on the floor?".

\subsection{Usability of HoloLens}

All the participants struggled with the weight of HL during the study. While most of them, except three, were happy to wear it continuously for approximately 40 minutes in this study, they expressed their reservations about wearing it for longer periods. They also noted the conspicuousness of HL as another factor that would prevent them from wearing it in public. "I won't wear this mainly because of what people would think of you. It's a vanity thing really" (P1) and "You wouldn't want to walk around in the street, unless you have people steering you to make it obvious that you are on a test" (P3). However, participants' opinions were split in terms of the screen size. Some felt that the current small screen size allowed them to use both the augmented view and their unaltered natural vision. "It (screen) didn't seem to be too small, too big, I still had peripheral vision. I knew where I was ... So, I didn't feel divorced from my surroundings which it is if you have VR goggles on" (P8) and "Instead of being all around you, it helps you to focus, you are focusing to a particular idea which is ideal as your eyes are moving... So that was good" (P6). However, some participants felt the screen size was too small. "If the view was bigger, that might help. As I don't have central vision, I have to move my eyes to find it" (P5) and "Screen size could be bigger, deeper. If it is wider, it's great, but a little deeper for me" (P3). Participants also liked being able to wear their prescription glasses underneath the HL in this study. While this study did not explore how visual augmentations were perceived in outdoor environments for all participants, two stood outside a building and observed the environment through HL during an overcast day and reported that they were able to perceive augmentations with ease.

\section{DISCUSSION AND IMPLICATIONS}

In the following sections, we discuss how different augmentations are perceived by people with different visual abilities, the subjective benefits and drawbacks participants experienced, and their requirements and suggestions in developing visual mobility aids for improved usability amongst a wider low vision population. Subsequently we discuss the implications of the results for future AR based O\&M aid designs.

\subsection{Residual vision and preference of visual augmentations}

One of the goals of this study was to explore the correlations between underlying visual ability and the types of augmentations people with LV find useful. It appeared that visual augmentations mainly benefited participants with VA better than 2/60. Participants with VA worse than 2/60 or with central vision loss were more likely to struggle to see the augmentations.

Participants with VA better than 2/60 liked the navigator prototype and live-view overlays. But for those with good VA and CS, the live-view overlays caused some blurring and double vision. Most of the participants also liked the idea of having an assistant helping to recognise things for them, except those with the best and worst residual vision in the study. The future studies should explore how the usability of automatic recognition could be improved by incorporating text and number reading (signs and buses) and automatic face recognition.

There was little interest in using colours to convey distance in prototypes 2 and 3, due to the limited colour perception in people with LV, and the significantly modified view of the world making it difficult for users to assimilate what they were seeing. 
For prototypes 1, 4, and 5, participants who liked these prototypes did not conform to any specific group of residual vision levels. Their differences in preferences for these prototypes seemed to stem from the differences in personality rather than vision. Their different expectations in technology played an important role in deciding the usefulness of different visual augmentations. For example, despite being able to see more via the HL than what she normally could with her residual vision, P1 did not find any of the prototypes useful because she was not able to recognise what she was seeing. However, P4 found most of the prototypes to be useful as they enabled him to see just enough to know things were around, without being able to recognise or identify what they were. As the preferences, expectations, and techniques on using residual vision differ from one person with LV to another, it is unlikely that one solution will fit all. To accommodate the needs of individuals, future designers of O\&M aids should provide a range of customisable visual augmentations and allow users to adjust and combine them based on their individual needs similar to the prescription of traditional low vision aids in low vision clinics which are tailored to the needs of the individual [21]. These visual augmentations should be adjustable in terms of their sizes, colours, placement.

Another factor limiting the potential usefulness of visual aids was the limited screen size of the glasses. Participants with severe central vision loss or patchy vision, e.g., P5 with severe RP, did not benefit from glasses as the visual augmentations were rendered in their blind spot. Therefore, future visual aids should consider screen sizes and anticipated user group. Designers should also consider implementing techniques such as warping and remapping [68] to display the visual augmentations in the good parts of the field of vision. In the future studies, it would also be useful to understand how similar visual augmentations are perceived by people with LV in video-seethrough display with larger screens (e.g., Oculus) compared to optical see-through displays.

\subsection{Benefits and drawbacks of visual augmentations}

In agreement with existing literature, all the different visualisation techniques from prototypes 1 - 6 improved participants' ability to perceive obstacles in different ways. This is crucial in achieving safe mobility as bumping into obstacles is one of the major problems experienced by people with LV. To maximise this benefit, designers of future studies should focus on implementing robust real-time obstacle detection algorithms that are accurate enough to pick up small tripping hazards, changes in surface levels, curbs, stairs and more [106], and visualise them. Additionally, as participants showed a strong preference for the navigator aid, there is a design opportunity to provide the visual navigation cues in conjunction with visualisations for obstacle detection to provide a holistic visual mobility aid (i.e., wayfinding and safety).

Another benefit of the augmentations from prototypes 4-6 was the improved face and facial feature recognition. These types of augmentations have the potential to improve participants' social life as lack of face recognition by people with LV is often a severe barrier in their social interactions [16]. The filtered-image overlays allowed them to see not only faces but also facial features and emotions (e.g., smiling). Additionally, they also enhanced visual details and allowed some participants to see what was in photos and paintings in their environment. This improved detail perception could open a design opportunity for exploring how people with LV can access arts visually, albeit altered to some extent, whereas their access to arts is limited to voice descriptions or tactile representation [10]. Additionally, future designers of visual aids should implement a wide range of image filters and allow users to combine them and fine-tune their parameters to suit the individual's needs. 
Although prototype 5 allowed many participants to see things in greater detail, displaying 2D high-contrast image overlays reduced depth perception for them. Future studies should explore ways to incorporate distance information in the edge overlays, perhaps colour-coding the edges or audio feedback.

One of the drawbacks of prototypes, as reported by participants was occlusion of the real-world environment by the visual augmentations. Despite being able to see obstacles and distances better with visual augmentations, many participants felt uncertain, and they wanted to be able to see real-world objects at times to better understand what they were seeing. This suggests future designs to incorporate efficient means of switching augmentations on and off to combine the benefits of both normal and augmented views.

Prototypes 2, 3, and 5 produced too much light for some participants and caused discomfort and difficulty seeing the enhancements until they manually reduced the screen brightness. As people with LV have difficulty adjusting to different light levels, future visual aids should be designed to maintain a consistent and comfortable level of brightness across different environments.

Another drawback of the augmentations in HL was difficulty perceiving colours correctly due to some participants experiencing diminished colour perception. To overcome this problem, designers of future aids should use fewer distinctive colours and should incorporate colour schemes that can be adjusted according to users' needs.

The important technical issue observed in prototypes 1,2, and 3 was slow and sparse depth maps and spatial updates from HL causing visible holes, colour specks in the visual augmentations and inability to deal with dynamic elements in the environment. More powerful hardware in the future could potentially alleviate these problems, but future studies could consider using additional data sources to improve efficiency and accuracy [25]. Another important technical issue is image stability for prototypes 4, 5, and 6. As even the slightest head movements alter the extracted edges, the final overlaid images look different from frame to frame, leading to jumpy and perpetually changing lines in some parts of the overlays. Other problems with image overlays included misaligned and blurry images, and delays in overlays. Designers of future aids should implement image stabilisation algorithms and improve image registration.

\section{LIMITATIONS}

While one of the strengths of this study was the diverse sample of participants with respect to residual vision, a limitation was the lack of proper representation of the most prevalent visual conditions such as macular degeneration and glaucoma. Another limitation was that because participants were recruited via low vision charities, they were active and engaging members of the low vision community who might not be representative of people with low vision more generally i.e. there was some potential for selection bias. Having long term vision loss and being well-adjusted to it physically and mentally, their feedback and opinions of the visual augmentations might be different from the majority of people with LV who enjoy less independence in their day-to-day lives. Another limitation of this study was the lack of a comprehensive evaluation of outdoor environments.

Regarding the technological aspects, limitations of the HoloLens as visual mobility aid included slow and sparse depth mapping, limited camera, small screen sizes, weight, and cost. Future technical improvements in these areas would significantly improve the usability of AR glasses for this vulnerable user group.

\section{CONCLUSION}

This study provides insights about user experiences with various visual augmentations and further highlights the diversity of low vision population. Based on the detailed qualitative data, we have made suggestions to improve the 
design and usability of visual augmentations as $0 \& \mathrm{M}$ aids. As people with low vision have highly varied visual needs and preferences, the ability to customise the augmentations to accommodate their needs will be critical for the success of any future visual low vision aids. The results also suggest that participants with VA better than 2/60 could benefit the most from visual augmentation.

Despite having some technical limitations (e.g., slow depth sensor, poor camera, unstable images) and limited customizability, many of the visual augmentations on HL were generally perceived as useful. With future advancements in hardware and software, AR glasses have the potential to deliver useful information visually and audibly as both standalone and complementary O\&M aids.

\section{Acknowledgement}

We thank all the participants and their helpers for their time and effort to participate in our study. We also would like to thank Sight Life, Sight Cymru, and Cardiff University low vision clinic for their support in recruitment and providing the locations to conduct the interviews.

\section{References}

[1] Dragan Ahmetovic, Cole Gleason, Chengxiong Ruan, Kris Kitani, Hironobu Takagi, and Chieko Asakawa. 2016. NavCog: A Navigational Cognitive Assistant for the Blind. Proc. 18th Int. Conf. Human-Computer Interact. with Mob. Devices Serv. - MobileHCI '16 (2016), 90-99. DOI:https://doi.org/10.1145/2935334.2935361

[2] Saleh Alghamdi, Ron Van Schyndel, and Ahmed Alahmadi. 2013. Indoor navigational aid using active RFID and QR-code for sighted and blind people. IEEE 8th Int. Conf. Intell. Sensors, Sens. Networks Inf. Process. 1, (2013), 18-22. DOI:https://doi.org/10.1109/ISSNIP.2013.6529756

[3] N Amin and M Borschbach. 2014. Classification criteria for local navigation digital assistance techniques for the visually impaired. In 2014 13th International Conference on Control Automation Robotics and Vision, ICARCV 2014, 1724-1728. DOI:https://doi.org/10.1109/ICARCV.2014.7064576

[4] B. Ando, S. Baglio, V. Marletta, and A. Valastro. 2015. A Haptic Solution to Assist Visually Impaired in Mobility Tasks. IEEE Trans. Human-Machine Syst. 45, 5 (2015), 641-646.

DOI:https://doi.org/10.1109/THMS.2015.2419256

[5] Anastasios Nikolas Angelopoulos, Hossein Ameri, Debbie Mitra, and Mark Humayun. 2019. Enhanced Depth Navigation Through Augmented Reality Depth Mapping in Patients with Low Vision. Sci. Rep. 9, 1 (2019), 11230. DOI:https://doi.org/10.1038/s41598-019-47397-w

[6] Henry Apfelbaum and Eli Peli. 2015. Tunnel Vision Prismatic Field Expansion: Challenges and Requirements. Transl. Vis. Sci. Technol. 4, 6 (2015), 8.

[7] Malika Auvray, Sylvain Hanneton, and J. Kevin O’Regan. 2007. Learning to perceive with a visuo-auditory substitution system: Localisation and object recognition with “The vOICe." Perception 36, 3 (2007), 416430. DOI:https://doi.org/10.1068/p5631

[8] Mauro Avila Soto, Markus Funk, Matthias Hoppe, Robin Boldt, Katrin Wolf, and Niels Henze. 2017. DroneNavigator. Proc. 19th Int. ACM SIGACCESS Conf. Comput. Access. - ASSETS '17 October (2017), 300304. DOI:https://doi.org/10.1145/3132525.3132556

[9] Jinqiang Bai, Shiguo Lian, Zhaoxiang Liu, Kai Wang, and Dijun Liu. 2017. Smart Guiding Glasses for Visually Impaired People in Indoor Environment. IEEE Trans. Consum. Electron. 63, 3 (August 2017), 258266.

[10] Jorge Iranzo Bartolome, Luis Cavazos Quero, Sunhee Kim, Myung Yong Um, and Jundong Cho. 2019. Exploring aRt with a voice controlled multimodal guide for blind people. In TEI 2019 - Proceedings of the 
13th International Conference on Tangible, Embedded, and Embodied Interaction, 383-390. DOI:https://doi.org/10.1145/3294109.3300994

[11] Alex Black, Jan E. Lovie-Kitchin, Russell L. Woods, Nicole Arnold, John Byrnes, and Jane Murrish. 1997. Mobility performance with retinitis pigmentosa. Clin. Exp. Optom. 80, 1 (1997), 1-12.

DOI:https://doi.org/10.1111/j.1444-0938.1997.tb04841.x

[12] B.B. Blasch, S.J. LaGrow, and De L'Aune. 1996. Three aspects of coverage provided by the long cane: Object, surface, and foot-placement preview. Journal of Visual Impairment \& Blindness.

[13] Johann Borenstein and Iwan Ulrich. 2001. Applying Mobile Robot Technologies to Assist the Visual Impaired. Guid. 31, 2 (2001), 131-136.

[14] Alex R. Bowers, Gang Luo, Noa M. Rensing, and Eli Peli. 2004. Evaluation of a prototype Minified Augmented-View device for patients with impaired night vision. Ophthalmic Physiol. Opt. 24, 4 (2004), 296-312.

[15] Michael Brock and Per Ola Kristensson. 2013. Supporting blind navigation using depth sensing and sonification. (2013), 255-258. DOI:https://doi.org/10.1145/2494091.2494173

[16] Diana M. Brouwer, Gaynor Sadlo, Karen Winding, and Marianne I.G. Hanneman. 2008. Limitations in mobility: Experiences of visually impaired older people. Br. J. Occup. Ther. 71, 10 (2008), 414-421.

[17] Stefano Burigat and Luca Chittaro. 2012. Mobile Navigation and Information Services for Disabled Students in University Buildings : a Needs Assessment Investigation. Proc. 2nd Work. Mob. Access. Mob. HCI 2012 Conf. (2012). Retrieved from https://pdfs.semanticscholar.org/611b/fa3b2cad2cec73cbfcb35a0b15b399449d55.pdf

[18] Sylvain Cardin, Daniel Thalmann, and Frederic Vexo. 2007. A wearable system for mobility improvement of visually impaired people. Vis. Comput. 23, 2 (February 2007), 109-118.

DOI:https://doi.org/10.1007/s00371-006-0032-4

[19] Ruiqi Cheng, Kaiwei Wang, Kailun Yang, Ningbo Long, Weijian Hu, Hao Chen, Jian Bai, and Dong Liu. 2017. Crosswalk navigation for people with visual impairments on a wearable device. J. Electron. Imaging 26, 5 (September 2017). DOI:https://doi.org/10.1117/1.JEI.26.5.053025

[20] Anne Lesley Corn and Jane N. Erin. 2010. Foundations of Low Vision: Clinical and Functional Perspectives.

[21] Michael D. Crossland and Janet H. Silver. 2005. Thirty years in an urban low vision clinic: Changes in prescribing habits of low vision practitioners. Optom. Vis. Sci. 82, 7 (2005), 617-622.

DOI:https://doi.org/10.1097/01.opx.0000171336.40273.3f

[22] Enox Software. 2017. OpenCV for Unity. Unity Asset Store. Retrieved from https://assetstore.unity.com/packages/tools/integration/opencv-for-unity-21088

[23] S Ertan, G Lee, A Willets, H Tan, and A Pentland. 1998. A wearable haptic navigation guidance system. In SECOND INTERNATIONAL SYMPOSIUM ON WEARABLE COMPUTERS - DIGEST OF PAPERS, 164-165. DOI:https://doi.org/10.1109/ISWC.1998.729547

[24] M R Everingham, B T Thomas, and T Troscianko. 1999. Head-mounted mobility aid for low vision using scene classification techniques. Int. J. Virtual Real. 3, 4 (1999), 3-12.

[25] Mathieu Garon, Pierre Olivier Boulet, Jean Philippe Doironz, Luc Beaulieu, and Jean Francois Lalonde. 2017. Real-Time High Resolution 3D Data on the HoloLens. Adjun. Proc. 2016 IEEE Int. Symp. Mix. Augment. Reality, ISMAR-Adjunct 2016 October (2017), 189-191. DOI:https://doi.org/10.1109/ISMARAdjunct.2016.0073

[26] João Guerreiro, Dragan Ahmetovic, Daisuke Sato, Kris Kitani, and Chieko Asakawa. 2019. Airport Accessibility and Navigation Assistance for People with Visual Impairments. (2019), 1-14. DOI:https://doi.org/10.1145/3290605.3300246

[27] Shirin E Hassan, John C Hicks, Hao Lei, and Kathleen A Turano. 2007. What is the minimum field of view required for efficient navigation? Vision Res. 47, 16 (July 2007), 2115-2123. 
DOI:https://doi.org/10.1016/j.visres.2007.03.012

[28] Jennifer B. Hassell, E. L. Lamoureux, and J. E. Keeffe. 2006. Impact of age related macular degeneration on quality of life. Br. J. Ophthalmol. 90, 5 (2006), 593-596.

[29] Else M. Havik, Aart C. Kooijman, and Frank J. J. M. Steyvers. 2019. The Effectiveness of Verbal Information Provided by Electronic Travel Aids for Visually Impaired Persons. J. Vis. Impair. Blind. 105, 10 (2019), 624-637. DOI:https://doi.org/10.1177/0145482x1110501009

[30] S L Hicks, I Wilson, L Muhammed, J Worsfold, S M Downes, and C Kennard. 2013. A Depth-Based HeadMounted Visual Display to Aid Navigation in Partially Sighted Individuals. PLoS One 8,7 (2013).

[31] A Hinds, A Sinclair, J Park, A Suttie, H Paterson, and M Macdonald. 2003. Impact of an interdisciplinary low vision service on the quality of life of low vision patients. Br. J. Ophthalmol. 87, 11 (2003), 1391-1396.

[32] Hein Min Htike, Tom H. Margrain, Yu Kun Lai, and Parisa Eslambolchilar. 2020. Ability of head-mounted display technology to improve mobility in people with low vision: A systematic review. Transl. Vis. Sci. Technol. 9, 10 (2020), 1-27. DOI:https://doi.org/10.1167/tvst.9.10.26

[33] Jonathan Huang, Max Kinateder, Matt J. Dunn, Wojciech Jarosz, Xing Dong Yang, and Emily A. Cooper. 2019. An augmented reality sign-reading assistant for users with reduced vision. PLoS One 14, 1 (2019), 1-9. DOI:https://doi.org/10.1371/journal.pone.0210630

[34] Alex D Hwang and Eli Peli. 2014. An Augmented-Reality Edge Enhancement Application for Google Glass. Optom. Vis. Sci. 91, 8 (2014), 1021-1030.

[35] Y Ikeda, E Suzuki, T Kuramata, T Kozaki, T Koyama, Y Kato, Y Murakami, H Enaida, and T Ishibashi. 2015. Development and evaluation of a visual aid using see-through display for patients with retinitis pigmentosa. Jpn. J. Ophthalmol. 59, 1 (2015), 43-47.

[36] Volodymyr Ivanchenko, James Coughlan, and Huiying Shen. 2008. Crosswatch: a Camera Phone System for Orienting Visually Impaired Pedestrians at Traffic Intersections. Comput Help People Spec Needs 5105, (2008), 1122-1128. DOI:https://doi.org/10.1007/978-3-642-31522-0

[37] William Henry Jacobson. 1993. The art and science of teaching orientation and mobility to persons with visual impairments. AFB Press.

[38] Rabia Jafri, Rodrigo Louzada Campos, Syed Abid Ali, and Hamid R Arabnia. 2018. Visual and Infrared Sensor Data-Based Obstacle Detection for the Visually Impaired Using the Google Project Tango Tablet Development Kit and the Unity Engine. IEEE ACCESS 6, (2018), 443-454.

DOI:https://doi.org/10.1109/ACCESS.2017.2766579

[39] Rabia Jafri and Marwa Mahmoud Khan. 2018. User-centered design of a depth data based obstacle detection and avoidance system for the visually impaired. HUMAN-CENTRIC Comput. Inf. Sci. 8, (May 2018). DOI:https://doi.org/10.1186/s13673-018-0134-9

[40] T Jones and To M Troscianko. 2006. Mobility performance of low-vision adults using an electronic mobility aid. Clin. Exp. Optom. 89, 1 (2006), 10-17.

[41] Hernisa Kacorri, Sergio Mascetti, Andrea Gerino, Dragan Ahmetovic, Valeria Alampi, Hironobu Takagi, and Chieko Asakawa. 2018. Insights on assistive orientation and mobility of people with visual impairment based on large-scale longitudinal data. ACM Trans. Access. Comput. 11, 1 (2018). DOI:https://doi.org/10.1145/3178853

[42] Jon S. Karlsson. 1998. Self-reports of psychological distress in connection with various degrees of visual impairment. J. Vis. Impair. Blind. 92, 7 (1998), 483-490.

DOI:https://doi.org/10.1177/0145482x9809200708

[43] Seita Kayukawa and Kris Kitani. 2019. BBeep: A Sonic Collision Avoidance System for Blind Travellers and Nearby Pedestrians. Proc. 2019 CHI Conf. Hum. Factors Comput. Syst. (2019), 1-12.

[44] Max Kinateder, Justin Gualtieri, Matt J. Dunn, Wojciech Jarosz, Xing-Dong Yang, and Emily A. Cooper. 2018. Using an Augmented Reality Device as a Distance-based Vision Aid-Promise and Limitations. Optom. Vis. 
Sci. 95, 9 (2018), 727-737.

[45] Eunjeong Ko and Eun Yi Kim. 2017. A Vision-Based Wayfinding System for Visually Impaired People Using Situation Awareness and Activity-Based Instructions. SENSORS 17, 8 (August 2017).

DOI:https://doi.org/10.3390/s17081882

[46] T Kuyk, JL Elliott, and PS Fuhr. 1998. Visual correlates of obstacle avoidance in adults with low vision. Optom. Vis. Sci. 75, 3 (1998), 174-82.

[47] T Kuyk, JL Elliott, and PS Fuhr. 1998. Visual correlates of mobility in real world settings in older adults with low vision. Optom. Vis. Sci. 75, 7 (1998), 538-547.

[48] Thomas Kuyk and Jeffry L. Elliott. 1999. Visual factors and mobility in persons with age-related macular degeneration. J. Rehabil. Res. Dev. 36, 4 (1999), 303-312.

[49] Ecosse L. Lamoureux, Jennifer B. Hassell, and Jill E. Keeffee. 2003. The determinants of participation in activities of daily living in people with impaired vision. Am. J. Ophthalmol. 137, 2 (2003), 265-270.

[50] Ecosse L. Lamoureux, Julie F. Pallant, Konrad Pesudovs, Jennifer B. Hassell, and Jill E. Keeffe. 2006. The impact of vision impairment questionnaire: An evaluation of its measurement properties using Rasch analysis. Investig. Ophthalmol. Vis. Sci. 47, 11 (2006), 4732-4741.

[51] Emma Marie Lethbridge and Chris Muldoon. 2018. Development of a Mobility-Related Quality-of-Life Measure for Individuals with Vision Impairments. J. Vis. Impair. Blind. 112, 2 (2018), 169-181.

[52] Claudio Loconsole, Maryam Banitalebi Dehkordi, Edoardo Sotgiu, Marco Fontana, Massimo Bergamasco, and Antonio Frisoli. 2016. An IMU and RFID-based Navigation System Providing Vibrotactile Feedback for Visually Impaired People. In EuroHaptics.

[53] Jan E. Lovie-Kitchin, Grace P. Soong, Shirin E. Hassan, and Russell L. Woods. 2010. Visual field size criteria for mobility rehabilitation referral. Optom. Vis. Sci. 87, 12 (2010), 948-957.

DOI:https://doi.org/10.1097/OPX.0b013e3181ff99be

[54] John B Lowe and Martin P Rubenstein. 2000. Distant telescopes: A survey of user success. Optom. Vis. Sci. 77, 5 (2000), 260-269. Retrieved from http://www.ncbi.nlm.nih.gov/htbinpost/Entrez/query?db=m\&form=6\&dopt=r\&uid=10831216

[55] Gang Luo and Eli; Peli. 2006. Use of an Augmented-Vision Device for Visual Search by Patients with Tunnel Vision. Invest. Ophthalmol. Vis. Sci. 47, 9 (2006), 4152-4159.

[56] Gang Luo and Eli Peli. 2011. Development and evaluation of vision rehabilitation devices. In IEEE Engineering in Medicine and Biology Society, EMBS, 5228-5231.

[57] Gang Luo, Russell L Woods, and Eli Peli. 2009. Collision judgment when using an augmented-vision headmounted display device. Invest Ophthalmol Vis Sci 50, 9 (2009), 4509-4515.

[58] M M Maassen, E Schwaderer, B Heinrich, S Herberhold, P S Mauz, and F Dammann. 2004. Comparison of the implantability of electronic hearing devices in a virtual reality planning environment and in human temporal bones. Acta Otolaryngol. 124, 9 (2004), 1039-1045. DOI:https://doi.org/10.1080/00016480410017837

[59] Roberto Manduchi and Sri Kurniawan. 2011. Mobility-related accidents experienced by people with visual impairment. Insight Res. Pract. Vis. Impair. Blind. 4, 2 (2011), 44-54.

[60] James A. Marron and Ian L. Bailey. 1982. Visual factors and orientation-mobility performance. Am. J. Optom. Physiol. Opt. 59, 5 (1982), 413-426.

[61] Peter B. L. Meijer. 1992. An Experimental System for Audiory Image Representations.pdf. IEEE Trans. Biomed. Eng. 39, 2 (1992), 112-121.

[62] Mei Miao, Martin Spindler, and Gerhard. Weber. 2021. Requirements of Indoor Navigation System from Blind Users. In Conference: Information Quality in e-Health.

[63] Microsoft. HoloLens (1st gen) hardware. Retrieved from https://docs.microsoft.com/enus/hololens/hololens1-hardware 
[64] Microsoft. Spatial Mapping. Retrieved from https://docs.microsoft.com/en-us/windows/mixedreality/holograms-230

[65] Microsoft. Locatable Camera - Mixed Reality. Retrieved from https://docs.microsoft.com/enus/windows/mixed-reality/locatable-camera

[66] Microsoft Corporation. 2020. Mixed Reality Toolkit Documentation. Microsoft Corporation. Retrieved from https://microsoft.github.io/MixedRealityToolkit-

Unity/Documentation/GettingStartedWithTheMRTK.html

[67] Bogdan Mocanu, Ruxandra Tapu, and Titus Zaharia. 2016. When ultrasonic sensors and computer vision join forces for efficient obstacle detection and recognition. Sensors (Switzerland) 16, 11 (2016). DOI:https://doi.org/10.3390/s16111807

[68] Howard Moshtael, Tariq Aslam, Ian Underwood, and Baljean Dhillon. 2015. High Tech Aids Low Vision: A Review of Image Processing for the Visually Impaired. Transl. Vis. Sci. Technol. 4, 4 (July 2015), 6. DOI:https://doi.org/10.1167/tvst.4.4.6

[69] Dejing Ni, Aiguo Song, Lei Tian, Xiaonong Xu, and Danfeng Chen. 2015. A Walking Assistant Robotic System for the Visually Impaired Based on Computer Vision and Tactile Perception. Int. J. Soc. Robot. 7, 5 (November 2015), 617-628. DOI:https://doi.org/10.1007/s12369-015-0313-z

[70] OpenCV. Canny Edge Detection. Retrieved from https://docs.opencv.org/3.4.10/da/d22/tutorial_py_canny.html

[71] Hugo Paredes, Hugo Fernandes, Paulo Martins, and João Barroso. 2013. Gathering the users' needs in the development of assistive technology: A blind navigation system use case. Lect. Notes Comput. Sci. (including Subser. Lect. Notes Artif. Intell. Lect. Notes Bioinformatics) 8011 LNCS, PART 3 (2013), 79-88. DOI:https://doi.org/10.1007/978-3-642-39194-1-10

[72] M D Peláez-Coca, F Vargas-Martín, Sonia Mota, J Díaz, Eduardo Ros-Vidal, Maria Dolores Pelaez-Coca, Fernando Vargas-Martin, Sonia Mota, Javier Diaz, and Eduardo Ros-Vidal. 2009. A versatile optoelectronic aid for low vision patients. Ophthalmic Physiol. Opt. 29, 5 (2009), 565-572.

[73] Eli Peli, Gang Luo, Alex Bowers, and Noa Rensing. 2009. Development and Evaluation of Vision Multiplexing Devices for Vision Impairments. Int. J. Artif. Intell. Tools 18, 3 (2009), 365-378.

[74] E E Pissaloux, R Velazquez, and F Maingreaud. 2017. A New Framework for Cognitive Mobility of Visually Impaired Users in Using Tactile Device. IEEE Trans. Human-Machine Syst. 47, 6 (2017), 1040-1051. DOI:https://doi.org/10.1109/THMS.2017.2736888

[75] Pablo Alejandro Quiñones, Tammy C. Greene, Rayoung Yang, and Mark W. Newman. 2011. Supporting visually impaired navigation: A needs-finding study. Conf. Hum. Factors Comput. Syst. - Proc. May 2011 (2011), 1645-1650. DOI:https://doi.org/10.1145/1979742.1979822

[76] C Ramer, T Lichtenegger, J Sessner, M Landgraf, and J Franke. 2016. An Adaptive, Color Based Lane Detection of a Wearable Jogging Navigation System for Visually Impaired on Less Structured Paths. In 2016 6TH IEEE INTERNATIONAL CONFERENCE ON BIOMEDICAL ROBOTICS AND BIOMECHATRONICS (BIOROB) (Proceedings of the IEEE RAS-EMBS International Conference on Biomedical Robotics and Biomechatronics), 741-746.

[77] Joseph Redmon and Ali Farhadi. 2018. YOLOv3 : An Incremental Improvement. arXiv (2018).

[78] Joram J. van Rheede, Iain R. Wilson, Rose I. Qian, Susan M. Downes, Christopher Kennard, and Stephen L. Hicks. 2015. Improving Mobility Performance in Low Vision With a Distance-Based Representation of the Visual Scene. Investig. Ophthalmol. Vis. Sci. 56, 8 (2015), 4802-4809.

[79] RNIB. 2019. Key information and statistics on sight loss in the UK. RNIB. Retrieved January 4, 2021 from http://www.rnib.org.uk/knowledge-and-research-hub/key-information-and-statistics

[80] Leat S.J. and Lovie-Kitchin J.E. 2008. Visual Function, Visual Attention, and Mobility Performance in Low Vision. Optom. Vis. Sci. 85, 11 (2008), 1049-1056. 
[81] D Sato, U Oh, K Naito, H Takagi, K Kitani, and C Asakawa. 2017. NavCog3: An evaluation of a smartphonebased blindindoor navigation assistant with semantic features in a large-scale environment. In ASSETS 2017 - Proceedings of the 19th International ACM SIGACCESS Conference on Computers and Accessibility, 270-279. DOI:https://doi.org/10.1145/3132525.3132535

[82] Shraga Shoval, Johann Borenstein, and Yoram Koren. 1998. The NavBelt - A computerized travel aid for the blind based on mobile robotics technology. IEEE Trans. Biomed. Eng. 45, 11 (1998), 1376-1386. DOI:https://doi.org/10.1109/10.725334

[83] W. C.S.S. Simoes and V. F. De Lucena. 2016. Blind user wearable audio assistance for indoor navigation based on visual markers and ultrasonic obstacle detection. 2016 IEEE Int. Conf. Consum. Electron. ICCE 2016 (2016), 60-63. DOI:https://doi.org/10.1109/ICCE.2016.7430522

[84] Janet P. Szlyk, Gerald A. Fishman, Sandeep Grover, Beatrise I. Revelins, and Deborah J. Derlacki. 1998. Difficulty in performing everyday activities in patients with juvenile macular dystrophies: Comparison with patients with retinitis pigmentosa. Br. J. Ophthalmol. 82, 12 (1998), 1372-1376.

[85] Sarit Szpiro, Yuhang Zhao, and Shiri Azenkot. 2016. Finding a store, searching for a product: a study of daily challenges of low vision people. UbiComp '16 (2016), 61-72.

[86] Ruxandra Tapu, Bogdan Mocanu, and Titus Zaharia. 2015. Automatic Assistant for Better Mobility and Improved Cognition of Partially Sighted Persons. Adv. Electr. Comput. Eng. 15, 3 (2015), 45-52.

DOI:https://doi.org/10.4316/AECE.2015.03006

[87] Ruxandra Tapu, Bogdan Mocanu, and Titus Zaharia. 2015. ALICE: A smartphone assistant used to increase the mobility of visual impaired people. J. Ambient Intell. Smart Environ. 7, 5 (2015), 659-678.

DOI:https://doi.org/10.3233/AIS-150336

[88] Virgil Tiponut, Sabin Ionel, Catalin-Daniel Caleanu, and Ioan Lie. 2007. Improved version of an integrated environment for assisted movement of visually impaired. In Proceedings - 11th WSEAS International Conference on Systems (Electrical and Computer Engineering Series), 87-91.

[89] B.S. Tjan, P.J. Beckmann, R. Roy, N. Giudice, and G.E. Legge. 2006. Digital Sign System for Indoor Wayfinding for the Visually Impaired. (2006), 30-30. DOI:https://doi.org/10.1109/cvpr.2005.442

[90] Kathleen A. Turano, Aimee T. Broman, Karen Bandeen-Roche, Beatriz Munoz, Gary S. Rubin, and Sheila K. West. 2004. Association of visual field loss and mobility performance in older adults: Salisbury eye evaluation study. Optom. Vis. Sci. 81, 5 (2004), 298-307.

[91] Kathleen A. Turano, Duane R. Geruschat, Julie W. Stahl, and Robert W. Massof. 1999. Perceived visual ability for independent mobility in persons with retinitis pigmentosa. Investig. Ophthalmol. Vis. Sci. 40, 5 (1999), 865-877.

[92] Unity. Unity. Retrieved from https://unity.com/

[93] Unity. Spatial Mapping Renderer. Retrieved from https://docs.unity3d.com/2018.3/Documentation/Manual/SpatialMappingRenderer.html

[94] Unity. NavMesh. Retrieved from https://docs.unity3d.com/ScriptReference/AI.NavMesh.html

[95] Fernando Vargas-martín, E L I Peli, The Schepens, Boston Ep, Laboratorio De Óptica, and Departamento De Física. 2002. Augmented-View for Restricted Visual Field : Multiple Device Implementations. Optom. Vis. Sci. 79, 11 (2002), 715-723.

[96] Ramiro Velázquez, Edwige Pissaloux, Pedro Rodrigo, Miguel Carrasco, Nicola Giannoccaro, and Aimé LayEkuakille. 2018. An Outdoor Navigation System for Blind Pedestrians Using GPS and Tactile-Foot Feedback. Appl. Sci. 8, 4 (2018), 578. DOI:https://doi.org/10.3390/app8040578

[97] Gale R. Watson. 2001. Low vision in the geriatric population: Rehabilitation and management. Journal of the American Geriatrics Society 49, 317-330. DOI:https://doi.org/10.1046/j.1532-5415.2001.4930317.x

[98] Michele A. Williams, Caroline Galbraith, Shaun K. Kane, and Amy Hurst. 2014. "Just Let the Cane Hit It." (2014), 217-224. DOI:https://doi.org/10.1145/2661334.2661380 
[99] Michele A. Williams, Amy Hurst, and Shaun K. Kane. 2013. "Pray Before You Step out”: Describing Personal and Situational Blind Navigation Behaviors. Proc. 15th Int. ACM SIGACCESS Conf. Comput. Access. - ASSETS' 15 (2013), p28. DOI:https://doi.org/10.1145/2513383.2513449

[100] Cang Ye, Soonhac Hong, Xiangfei Qian, and Wei Wu. 2016. Co-Robotic Cane: A New Robotic Navigation Aid for the Visually Impaired. IEEE Syst. Man, Cybern. Mag. 2, 2 (2016), 33-42.

DOI:https://doi.org/10.1109/msmc.2015.2501167

[101] Yitzhak Yitzhaky and Liron Itan. 2013. Performance of visual tasks from contour information. J. Opt. Soc. Am. A, Opt. image Sci. Vis. 30, 3 (2013), 392-402.

[102] Ola Younis, Waleed Al-Nuaimy, Mohammad H. Alomari, and Fiona Rowe. 2019. A hazard detection and tracking system for people with peripheral vision loss using smart glasses and augmented reality. Int. J. Adv. Comput. Sci. Appl. 10, 2 (2019), 1-9. DOI:https://doi.org/10.14569/ijacsa.2019.0100201

[103] Yuhang Zhao, Michele Hu, Shafeka Hashash, and Shiri Azenkot. 2017. Understanding Low Vision People's Visual Perception on Commercial Augmented Reality Glasses. In Proceedings of the 2017 ACM SIGCHI Conference on Human Factors in Computing Systems (CHI'17), 4170-4181.

[104] Yuhang Zhao, Elizabeth Kupferstein, Brenda Veronica Castro, Steven Feiner, and Shiri Azenkot. 2019. Designing AR visualizations to facilitate stair navigation for people with low vision. UIST 2019 - Proc. 32nd Annu. ACM Symp. User Interface Softw. Technol. (2019), 387-402. DOI:https://doi.org/10.1145/3332165.3347906

[105] Yuhang Zhao, Elizabeth Kupferstein, Hathaitorn Rojnirun, Leah Findlater, and Shiri Azenkot. 2020. The Effectiveness of Visual and Audio Wayfinding Guidance on Smartglasses for People with Low Vision. (2020), 1-14. DOI:https://doi.org/10.1145/3313831.3376516

[106] Yuhang Zhao, Elizabeth Kupferstein, Doron Tal, and Shiri Azenkot. 2018. "It Looks Beautiful but Scary:" How Low Vision People Navigate Stairs and Other Surface Level Changes. Proc. 20th Int. ACM SIGACCESS Conf. Comput. Access. - ASSETS '18 (2018), 307-320. DOI:https://doi.org/10.1145/3234695.3236359

[107] Yuhang Zhao, Sarit Szpiro, Jonathan Knighten, and Shiri Azenkot. 2016. CueSee: Exploring Visual Cues for People with Low Vision to Facilitate a Visual Search Task. Proc. 2016 ACM Int. Jt. Conf. Pervasive Ubiquitous Comput. - UbiComp '16 (2016), 73-84.

[108] Yuhang Zhao, Cornell Tech, and Cornell Tech. 2015. ForeSee: A Customizable Head-Mounted Vision Enhancement System for People with Low Vision. In ASSETS '15: Proceedings of the 17th International ACM SIGACCESS Conference on Computers \& Accessibility, 239-249. 